\title{
A pre-shaped guidewire could cause left ventricular perforation during transcatheter aortic valve implantation
}

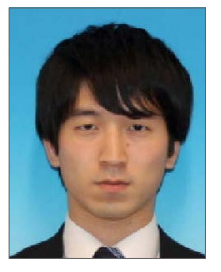

Ryo Abe1, MD; Ryosuke Higuchi1*, MD; Atsushi Shimizu²,3, MD; Mike Saji ${ }^{1}$, MD;

Itaru Takamisawa ${ }^{1}, \mathrm{MD}$

1. Department of Cardiology, Sakakibara Heart Institute, Tokyo, Japan; 2. Department of Cardiovascular Surgery, Sakakibara Heart Institute, Tokyo, Japan; 3. Department of Cardiovascular Surgery, Saitama Sekishinkai Hospital, Saitama, Japan

This paper also includes supplementary data published online at: https://www.asiaintervention.org/doi/10.4244/AIJ-D-21-00025

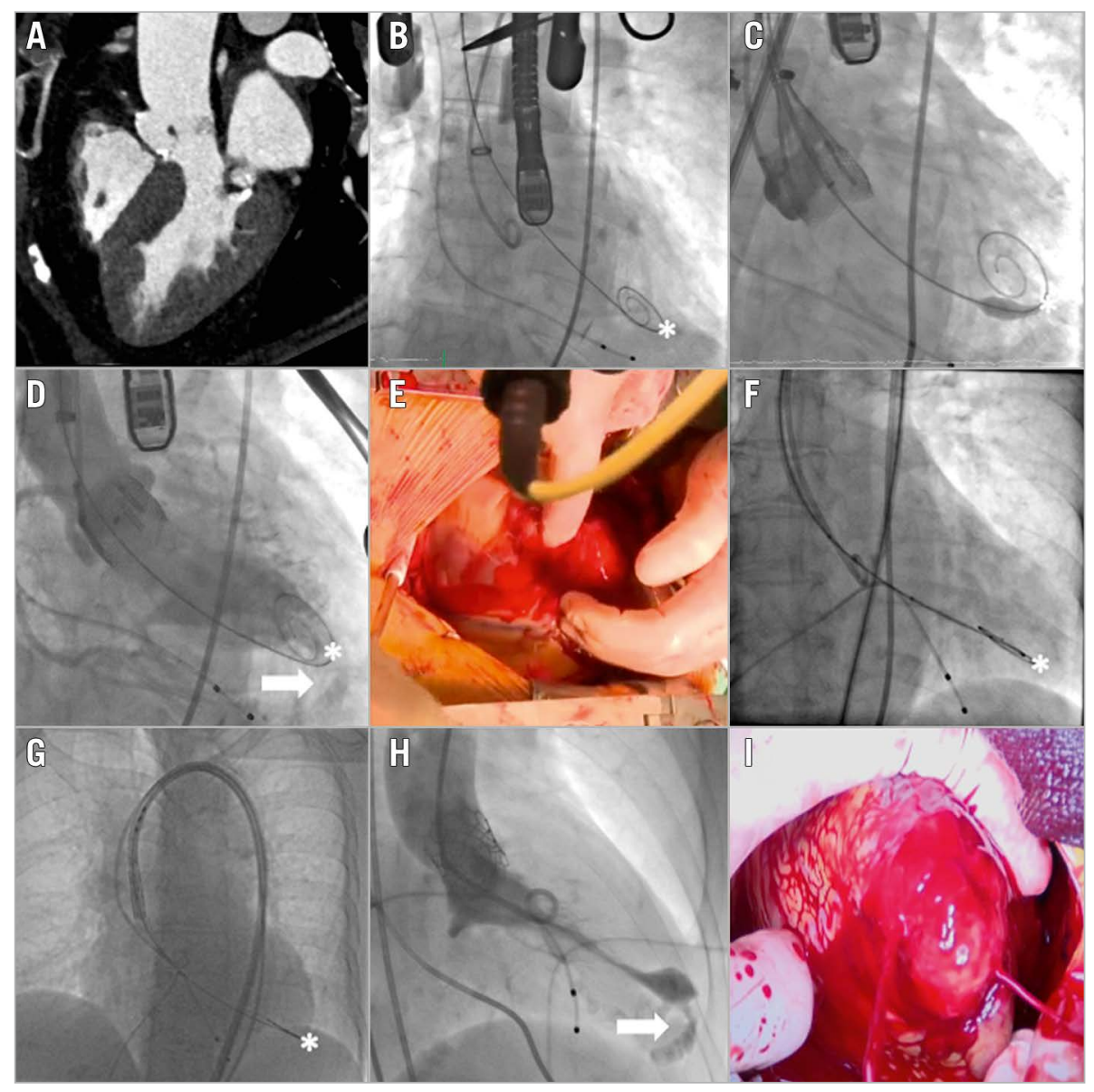

*Corresponding author: Department of Cardiology, Sakakibara Heart Institute, Fuchu, Asahicho, 3-chome-16-1, Tokyo, Japan. E-mail:rhiguchi@shi.heart.or.jp 
Life-threatening complications may occur even in contemporary transcatheter aortic valve implantation (TAVI). A pre-shaped guidewire, instead of a manually shaped guidewire, is thought to prevent left ventricular (LV) perforation; however, we experienced two cases of LV perforation using a pre-shaped guidewire.

Case 1 was a 90-year-old female with severe aortic stenosis (Panel A). A transaortic approach was selected due to the small iliac artery; this was the second transaortic TAVI performed at our facility. A SAFARI ${ }^{2 T M}$ (Boston Scientific, Marlborough, MA, USA) pre-shaped transcatheter aortic valve replacement (TAVR) guidewire was placed at the LV apex, and a $21 \mathrm{~mm}$ LOTUS Edge ${ }^{\mathrm{TM}}$ aortic valve (Boston Scientific) was positioned (Panel B; *: the tip of the guidewire). Blood pressure decreased after valve deployment, and aortography revealed a perforated LV apex (Panel C, Panel D; arrow: extravasation) (Moving image 1). Pericardiac drainage and cardiopulmonary bypass were immediately established, and emergent surgery with the David-Komeda technique was successfully performed (Panel E).

Case 2 was an 82-year-old female undergoing transfemoral TAVI. This was the seventh TAVI performed by the first operator; the second operator was the resident physician. A $23 \mathrm{~mm}$ SAPIEN 3 (Edwards Lifesciences, Irvine, CA, USA) transcatheter heart valve was delivered using a SAFARI ${ }^{2}$ pre-shaped TAVR guidewire placed at the LV apex (Panel F, Panel G). The patient's blood pressure decreased after valve deployment, and left ventriculography showed a perforation of the LV apex (Panel H, Moving image 2). Bail-out surgery was performed to repair the lacerated LV apex without major issues (Panel I).
The tip of the guidewire was transferred during valve positioning and delivery, and a forced guidewire caused LV perforation. The LV apex is only 1-2 mm thick, even in a hypertrophied ventricle. Lack of attention to the position of the guidewire, an insufficient level of experience, and the longer shaft of the LOTUS Edge could be the cause of LV perforation. The placement of a guidewire at the posterior site, and optimal size selection might prevent LV perforation. LV perforation can occur even when using a pre-shaped guidewire. Close attention and adequate handling of the guidewire is, therefore, essential.

\section{Acknowledgements}

The authors wish to thank all the members of the Heart Valve Team.

\section{Conflict of interest statement}

I. Takamisawa is a clinical proctor of TAVI for Edwards Lifesciences and Medtronic. The other authors have no conflicts of interest to declare.

\section{Supplementary data}

Moving image 1. Aortography following valve deployment in case 1 .

Moving image 2. Left ventriculography following valve deployment in case 2.

The supplementary data are published online at:

https://www.asiaintervention.org/

doi/10.4244/AIJ-D-21-00025 